\title{
The role of MR-PET in abdominal and pelvic oncology
}

\author{
Julia R. Fielding \\ Department of Radiology, University of North Carolina at Chapel Hill, Chapel Hill, NC 27599, USA
}

MR-PET, developed over the past 5 years, is a new tool for identifying and staging cancer treatment. Similar to CT-PET, functional images are superimposed over static images. Despite the complexity of the equipment, MRPET is now produced by several vendors. It is in place in multiple sites in Europe and North America. In retrospective studies, MR-PET was equal in diagnostic accuracy to CT-PET. It has been reported superior to CT-PET in identification of liver metastases, malignant bone lesions, and accurate assessment of $\mathrm{T}$ stage in a mixed tumor population. Large prospective studies have not yet been performed. MR-PET is of particular value in children and young women to avoid ionizing radiation.

MR-PET images may be acquired simultaneously using a fused system or sequentially with tables in adjacent rooms. In general, a full-body PET is performed with superimposed 3D gradient echo images with and without administration of Gd chelates. Diffusion images are often performed to determine the activity of small lesions. Additional pulse sequences and imaging planes in tandem with Gd chelates and novel PET tracers provide excellent anatomic and functional imaging in the brain, retroperitoneum, and pelvis.

Fusion of ultra-fast MR sequences and PET images obtained during quiet breathing and bone artifact remains problematic. Multiple PET beds may fail to include the organ of interest in the center of the magnetic field. Research continues to resolve these problems.

This issue is designed to provide an introduction to the basic physics behind PET/MR, review current literature focusing on diagnosis and staging of cancer, and give advice on implementation of the technology into a busy practice. 